\section{MS2-P3 Developing and optimizing serial crystallography for static and dynamic structural biology}

Dominik Oberthür ${ }^{1}$, Juraj Knoska ${ }^{1,2}$, Max Wiedorn ${ }^{1,2}$, Carolin Seuring ${ }^{1,3}$, Kenneth Beyerlein $^{1}$, Lars Gumprecht ${ }^{1}$, Anton Barty ${ }^{1}$, Alke Meents ${ }^{1}$, Sasa Bajt ${ }^{4}$, Henry N. Chapman ${ }^{1,2,3}$

1. Center for Free-Electron Laser Science, Deutsches Elektronen-Synchrotron DESY, Notkestraße 85, 22607 Hamburg, Germany

2. Department of Physics, University of Hamburg, Luruper Chaussee 149, 22761 Hamburg, Germany

3. Centre for Ultrafast Imaging, Luruper Chaussee 149, 22761 Hamburg, Germany

4. Photon Science, Deutsches Elektronen-Synchrotron DESY, Notkestraße 85, 22607 Hamburg, Germany

email: dominik.oberthuer@desy.de

In time resolved studies, the use of micron- or sub-micron sized protein crystals not only allows for uniform laser excitation of all unit-cells in the X-ray beam, but for mix-and-inject (Schmidt, 2013) studies of enzymatic reactions that cannot be photo-induced. The small dimensions allow for fast enough diffusion of substrate into the crystals, such that those reactions can be induced homogenously at a defined time delay to the $\mathrm{X}$-ray probe. This has strong implications for sample preparation: in case of such experiments one deliberately strives to grow micro- or even 'invisible' nano-crystals of proteins that would otherwise form larger crystals as well. Mix-and-inject experiments require furthermore a narrow size distribution of the crystals and fast mixing of the crystal suspension with substrate solution.

Serial crystallography methods, now established at both XFEL (Chapman, 2011) and third generation synchrotron sources (Gati, 2013; Stellato, 2014; Nogly, 2015) are very well suited for such time-resolved experiments (Tenboer, 2014; Barends, 2015), making it possible to reveal the dynamic nature of biological macromolecules and their interactions at near-atomic spatial resolution and on ultrafast timescales, even for extremely radiation sensitive samples.

Here we describe promising new crystallization methods for such experiments, the CFEL-pipeline for crystal characterization prior to the crystallographic experiment and novel sample delivery methods for reduced sample consumption, increased data collection efficiency and designed for mix-and-inject experiments.

Barends, T.R. et al., (2015) Direct observation of ultrafast collective motions in CO myoglobin upon ligand dissociation. Science 350, 445-50.

Chapman, H. N. et al., (2011) Femtosecond X-ray protein nanocrystallography. Nature 470, 73-77.

Gati, C. et al., (2014) Serial crystallography onin vivogrown microcrystals using synchrotron radiation. IUCrJ 1.

Nogly, P. et al. ,(2015)Lipidic cubic phase serial millisecond crystallography using synchrotron radiation. IUCrJ 2.

Schmidt, M., (2013) Mix and Inject. Advances in Condensed Matter Physics 2013, 1-10.

Stellato, F. et al., (2014) Room-temperature macromolecular serial crystallography using synchrotron radiation. IUCrJ 1, 204-212.
Tenboer, J. et al., (2014) Time-resolved serial crystallography captures high-resolution intermediates of photoactive yellow protein. Science 346, 1242-1246.

Keywords: Serial Crystallography, SFX, Structural Biology, Sample Preparation, Time Resolved 